\title{
MIR31HG promotes cell proliferation and invasion by activating the Wnt/及-catenin signaling pathway in non-small cell lung cancer
}

\author{
SHUAIYU ZHENG, XIAOJIN ZHANG, XIAN WANG and JIYUAN LI \\ Department of Thoracic Surgery, The First Affiliated Hospital and College of Clinical Medicine \\ of Henan University of Science and Technology, Luoyang, Henan 471003, P.R. China
}

Received April 28, 2017; Accepted May 18, 2018

DOI: $10.3892 / \mathrm{ol} .2018 .9607$

\begin{abstract}
Long non-coding RNAs (lncRNAs) have recently been demonstrated to serve crucial roles in various diseases including tumor initiation and progression. However, the role of the lncRNA MIR31HG in non-small cell lung cancer (NSCLC) was not well established. The present study demonstrated that MIR31HG was significantly increased in tumor tissues compared with adjacent normal tissues, and increased MIR31HG expression levels were associated with histological differentiation grade, lymph node metastasis and Tumor-node metastasis (TNM) stage in patients with NSCLC. Patients who had a higher MIR31HG expression level, were predicted a shorter over survival (OS) time. Using in vitro assays, the present study demonstrated that the downregulation of MIR31HG expression significantly inhibited cell proliferation and cell invasion abilities. Furthermore, it was identified that knockdown of MIR31HG expression suppressed the cell epithelial-mesenchymal transition (EMT) phenotype by reducing the expression levels of Twist1 and Vimentin, but also increased the expression level of E-cadherin in NSCLC cells. Furthermore, the results of the present study demonstrated that downregulated MIR31HG inhibited the Wnt/ $\beta$-catenin signaling pathway by decreasing the expression of glycogen synthase kinase $3 \beta(\mathrm{GSK} 3 \beta)$ and $\beta$-catenin, but increasing the phosphorylated (p)-GSK3 $\beta$ expression in NSCLC cells. Together, these data demonstrated that MIR31HG could be identified as a poor prognostic biomarker and a novel therapeutic target for patients with NSCLC.
\end{abstract}

Correspondence to: Dr Shuaiyu Zheng, Department of Thoracic Surgery, The First Affiliated Hospital and College of Clinical Medicine of Henan University of Science and Technology, 24 Jinghua Road, Luoyang, Henan 471003, P.R. China

E-mail: zhengshuaiyu12@126.com

Key words: non-small cell lung cancer, epithelial-mesenchymal transition, cell invasion

\section{Introduction}

Lung cancer is an extremely malignant human tumor and has a high cancer-associated morbidity worldwide (1). Non-small cell lung cancer (NSCLC) accounts for $>80 \%$ of lung cancer globally, including small squamous cell carcinoma (SCC), adenocarcinoma (AD) and large cell carcinoma (LCC), in 2015 (2). Despite significant advances in the presently available treatment methods including surgery combined with radiotherapy and/or chemotherapy, due to local recurrence and metastasis in the disease, the 5-year survival rate is poor $(1,3)$. Therefore, it is necessary to investigate biological markers with high specificity and sensitivity for early diagnosis and novel therapeutic targets for NSCLC.

Accumulating evidence have demonstrated that long non-coding RNAs (lncRNAs) function as regulators in a variety of diseases, including cancer, neurological disorders, and fragile X syndrome (4). Abnormal alterations in the function of lncRNAs in certain tumors were identified to either promote or suppress tumor formation, development, progression and metastasis (5). A number of lncRNAs, including H19 and Homeobox transcript antisense RNA, have potential clinical applications as prognostic markers or therapeutic targets in some tumors (6).

MIR31HG is a lncRNA that has been identified as $>2,166$ nucleotides in length (7). A previous study reported that inhibition of MIR31HG promoted a strong p16 (INK4A)-dependent senescence phenotype (7). Downregulation of lncRNA MIR31HG was associated with a shorter survival time in patients with gastric cancer and promoted cell proliferation (8). In bladder cancer, MIR31HG expression levels were revealed to be remarkably reduced and was negatively associated with Tumor-node metastasis (TNM) stage (9). The lncRNA MIR31HG exhibited oncogenic potential in pancreatic ductal adenocarcinoma and was negatively regulated by miR-193b in order to promote tumor progression (10). Recent studies showed that increased MIR31HG 1ncRNA expression increased gefitinib resistance in NSCLC lines through the EGFR/PI3K/AKT signaling pathway (11). However, the clinical role and biological function of MIR31HG involved in NSCLC was has not been completely investigated.

The present study demonstrated that MIR31HG was significantly increased in tumor tissues, and patients with higher a MIR31HG expression, were predicted a shorter 
overall survival (OS) time. Using in vitro assays, the downregulation of MIR31HG expression was demonstrated to significantly inhibit cell proliferation, invasion and the epithelial-mesenchymal transition (EMT) phenotype in NSCLC cells. Furthermore, downregulated MIR31HG inhibited the Wnt/ $\beta$-catenin signaling pathway. Taken together, these data demonstrated that MIR31HG could be identified as a poor prognostic biomarker and a novel therapeutic target for patients with NSCLC.

\section{Materials and methods}

Human tissue samples. Human NSCLC tissue and paired normal tissue samples were collected from 88 patients ( 49 males and 39 females; mean age, 50 years; range, 32-76 years) who underwent radical surgery at The First Affiliated Hospital and College of Clinical Medicine of Henan University of Science and Technology (Luoyang, China) between March 2007 and July 2012. No patient had received radiotherapy or chemotherapy prior to surgery. All tissue samples were immediately frozen in liquid nitrogen and stored at $-80^{\circ} \mathrm{C}$ until subsequent experimentation. The experimental protocol was conducted according to the principles of the Declaration of Helsinki and was approved by the Ethics Committee of The First Affiliated Hospital and College of Clinical Medicine of Henan University of Science and Technology (Luoyang, China). Written, informed consent was obtained from all patients. The TNM staging followed NSCLC TNM staging criteria of American Joint Committee on Cancer 2003 edition (12).

Cell culture. Three human NSCLC cell lines A549, H1299 and NCIH460 and a normal human bronchial epithelial cell line 16HBE were purchased from Cell Bank at Chinese Academy of Sciences (Shanghai, China). Cells were cultured in RPMI-1640 medium (Gibco; Thermo Fisher Scientific, Inc., Waltham, MA, USA) and were supplemented with $10 \%$ fetal bovine serum (FBS) and $100 \mathrm{U} / \mathrm{ml}$ penicillin and $100 \mu \mathrm{g} / \mathrm{ml}$ streptomycin (Thermo Fisher Scientific, Inc.). The cell lines were maintained at $37^{\circ} \mathrm{C}$ in a humidified atmosphere of $5 \%$ $\mathrm{CO}_{2}$.

Cell transfection. The small interfering (si)-negative control (NC), si-MIR31HG-1 and si-miR31HG-2 used in this study were synthesized by Ribobio (Guangzhou RiboBio Co., Ltd., Guangzhou, China). The following sequences were used: si-MIR31HG-1, sense, 5'-A AGAAUGUGUUGUGGACA CAA-3', and anti-sense, 5'-UUGUGUCCACAACACAUU CUU-3'. si-miR31HG-2, sense, 5'-AAUGGAGCACAAAUA GUUU-3', and anti-sense, 5'-AAACUAUUUGUGCUCCAU U-3'. si-NC, sense, 5'-UUCUCCGAACGUGUCACGUTT-3', and anti-sense, 5'-ACGUGACACGUUCGGAGAATT-3'. The cells were transfected with si-MIR31HG-1, si-miR31HG-2 or si-NC (100 nM, respectively) according to the manufacturer's protocol. Cells transfection was conducted using Lipofectamine 2000 reagents (Thermo Fisher Scientific, Inc.) according to the manufacturer's protocol. Cells were harvested following transfection at $48 \mathrm{~h}$.

Cell proliferation, cell colony formation and cell migration assays. A Cell Counting kit-8 (CCK-8) assay was performed to evaluate NCIH460 or A549 cell proliferation by using the CCK-8 assay kit (Beyotime Institute of Biotechnology, Haimen, China). Briefly, 5,000 cells/well were cultured on a 96-well plate. The si-NC, si-MIR31HG-1 and si-MIR31HG-2 were transfected into the cells using Lipofectamine 2000 according to the manufacturer's protocol, as described previously. After 1, 2, 3, 4 and 5 days of transfection with RPMI-1640 medium (Gibco; Thermo Fisher Scientific, Inc.) containing sterile CCK-8 dye (10 $\mu \mathrm{l})$ was added to each well, after which the cells were incubated at $37^{\circ} \mathrm{C}$ for a further $4 \mathrm{~h}$ and the absorbance at $450 \mathrm{~nm}$ was measured in a microtiter plate reader (Molecular Devices, LLC, Sunnyvale, CA, USA). For the cell colony formation assay, a total of 100 cells/well transfected with si-NC, si-MIR31HG-1 and si-MIR 31HG-2 were seeded into a 12-well plate and cultured for 2 weeks. Cells were then fixed with $4 \%$ paraformaldehyde for $10 \mathrm{~min}$ at room temperature and stained with $0.1 \%$ crystal violet for $10 \mathrm{~min}$ at room temperature. The cells were observed and calculated with an inverted microscope (IX71; Olympus Corporation, Tokyo, Japan, magnification, x200). For the cell migration assay, the cells invasive ability was measured using Transwell insert with $8.0 \mu \mathrm{m}$ pore polycarbonate membrane coated with Matrigel (BD Biosciences, Franklin Lakes, NJ, USA). Briefly, $1 \times 10^{5}$ cells/well transfected with si-NC, si-MIR31HG-1 and si-MIR31HG-2 were plated onto the upper chambers of the transwell coated with Matrigel in serum-free RPMI-1640 medium and the lower chambers of the transwell were added with RPMI-1640 supplemented with 10\% FBS. Following a $48 \mathrm{~h}$ incubation at room temperature, cells on the upper surface of the filter were removed using a cotton swab, then cells were fixed with $4 \%$ paraformaldehyde for $20 \mathrm{~min}$ at room temperature and stained with $0.1 \%$ crystal violet for $20 \mathrm{~min}$ at room temperature. The cells were observed and measured in 10 randomly selected fields using an inverted microscope (magnification x200; CKX41; Olympus Corporation; magnification, $\mathrm{x} 200$ ).

Reverse transcription-quantitative polymerase chain reaction (RT-qPCR) assay. Total RNA from tissues and NSCLC and 16HBE cells was purified by the TRIzol (Invitrogen; Thermo Fisher Scientific, Inc., Waltham, MA, USA) according to the manufacturer's protocol. RNA quality was determined by a Nanodrop spectrophotometer. The RNA was reverse-transcribed into cDNA using RevertAid First Strand cDNA Synthesis kit (catalog no. K1622; Thermo Fisher Scientific, Inc.), according to the manufacturer's protocol. The mRNA expression was detected using SYBR Green PCR Master Mix (Applied Biosystems; Thermo Fisher Scientific, Inc.), according to the manufacturer's protocol, on ABI7500 system (ABI7500; Thermo Fisher Scientific, Inc.). The thermocycling conditions were as follows: $95^{\circ} \mathrm{C}$ for $10 \mathrm{~min}$, followed by 40 cycles of $95^{\circ} \mathrm{C}$ for $15 \mathrm{sec}$ and $60^{\circ} \mathrm{C}$ for $30 \mathrm{sec}$. Relative mRNA expression was calculated via $2^{-\triangle \Delta C q}$ methods (13). RT-qPCR experiments were replicated at least three times. The PCR primer sequences were as follows: MIR31HG forward, 5'-CGCTTCTGTCCT CCTACTCG-3', and MIR31HG reverse, 5'-ACAAGCAGA CCCTTGGAATG-3'. GAPDH forward, 5'-ATGGGGAAG GTGAAGGTCG-3', and GAPDH reverse, 5'-GGGTCA TTGATGGCAACAATATC-3'. Vimentin forward, 5'-AGG 
Table I. Associations between MIR31HG expression and clinicopathological parameters in 88 cases of NSCLC patients.

\begin{tabular}{|c|c|c|c|c|}
\hline \multirow[b]{2}{*}{ Clinicopathologic parameters } & \multicolumn{4}{|c|}{ MIR31HG expression levels } \\
\hline & Patient no. & Lower $(n=41)$ & Higher (47) & P-value \\
\hline Age (years) & & & & 0.594 \\
\hline$\leq 60$ & 52 & 23 & 29 & \\
\hline$>60$ & 36 & 18 & 18 & \\
\hline Sex & & & & 0.224 \\
\hline Male & 49 & 20 & 29 & \\
\hline Female & 39 & 21 & 18 & \\
\hline Tumor size & & & & 0.876 \\
\hline$<3 \mathrm{~cm}$ & 48 & 22 & 26 & \\
\hline$>3 \mathrm{~cm}$ & 40 & 19 & 21 & \\
\hline Smoking & & & & 0.285 \\
\hline No & 44 & 23 & 21 & \\
\hline Yes & 44 & 18 & 26 & \\
\hline Histological differentiation grade & & & & $0.024^{\mathrm{a}}$ \\
\hline Moderate-well & 32 & 20 & 12 & \\
\hline Poorly & 56 & 21 & 35 & \\
\hline Histology & & & & 0.294 \\
\hline $\mathrm{SCC}$ & 55 & 28 & 27 & \\
\hline $\mathrm{AC}$ & 33 & 13 & 20 & \\
\hline Lymph node metastasis & & & & $0.021^{\mathrm{a}}$ \\
\hline Negative & 40 & 24 & 16 & \\
\hline Positive & 48 & 17 & 31 & \\
\hline TNM stage (12) & & & & $0.002^{\mathrm{a}}$ \\
\hline I-II & 49 & 30 & 19 & \\
\hline III & 39 & 11 & 28 & \\
\hline
\end{tabular}

TNM, tumor-node-metastasis staging system; SCC, small squamous cell carcinoma; AC, adenocarcinoma. ${ }^{\mathrm{a} P}<0.05$.

AATGGCTCGTCACCTTCGTGAATA-3' and Vimentin reverse, 5'-GGAGTGTCGGTTGTTAAGAACTAGAGC T-3'. Twist1 forward, 5'-CATGTCCGCGTCCCACTAG-3', and Twist1, reverse 5'-TGTCCATTTTCTCCTTCTCTCG-3'. E-cadherin forward, 5'-TAGAGGGTCACCGCGTCTAT-3', and E-cadherin reverse, 5'-CGTACCGCTGATTGGCTG AG-3'.

Western blot assays. NCIH460 and A549 cells were lysed in RIPA buffer (Beyotime Institute of Biotechnology, Shanghai, China) and concentrations of supernatant protein were determined using the BCA protein assay kit (Beyotime Institute of Biotechnology). Supernatant samples containing $40 \mu \mathrm{g}$ total proteins were separated by $10 \%$ sodium dodecylsulfate-polyacrylamide gel electrophoresis (SDS-PAGE), and then were transferred to nitrocellulose membrane (Merck KGaA, Darmstadt, Germany). After blocking with 5\% fat-free milk at room temperature for $1 \mathrm{~h}$, the membrane was incubated with anti-E-cadherin antibody (cat. no. sc-21791; 1:1,000), anti-Twist1 antibody (cat. no. sc-6070; 1:2,000), anti-Vimentin antibody (cat. no. sc-80975; 1:1,000), anti-glycogen synthase kinase $3 \beta$ (GSK3 $\beta$; cat. no. sc-53931; 1:2,000), anti- $\beta$-catenin (cat. no. sc-1496; 1:2,000), anti-p-GSK3 $\beta$ (cat. no. sc-373800; 1:2,000) and anti-GAPDH antibody (cat. no. 166574; 1:1,000 dilution) (all from Santa Cruz Biotechnology, Inc., Dallas, TX, USA) at $4^{\circ} \mathrm{C}$ overnight and then incubated with appropriate horseradish peroxidase-conjugated secondary antibodies (goat anti-rabbit; dilution, 1:5,000; cat. no. ab7832; Abcam, Cambridge, MA, USA) at room temperature for $2 \mathrm{~h}$ and visualized using Pierce Enhanced Chemiluminescent Western Blotting Substrate (Pierce; Thermo Fisher Scientific, Inc.). The intensity of the bands was quantified using Image $\mathrm{Lab}^{\mathrm{TM}}$ Software (version 4.6.9; Bio-Rad Laboratories, Inc., Hercules, CA, USA).

Statistical analysis. The data are presented as mean \pm standard deviation from at least three experiments. Statistical analysis was performed using SPSS version 11.0 for Windows (SPSS, Inc., Chicago, IL, USA). Comparisons of quantitative data were analyzed by Student's t-test between two groups (two-tailed). One-way analysis of variance (ANOVA) for comparisons of multiple groups and Student Newman-Keuls was used as a post hoc test following ANOVA. $\mathrm{P}<0.05$ was considered to indicate a statistically significant difference. 
A

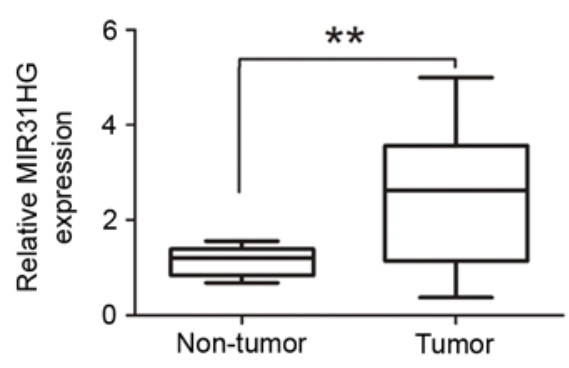

C

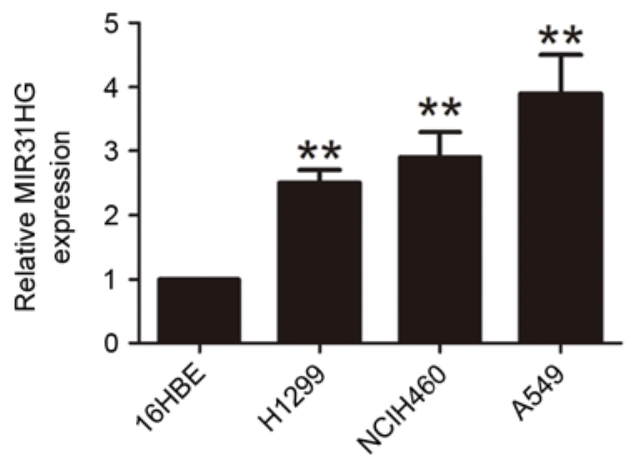

B
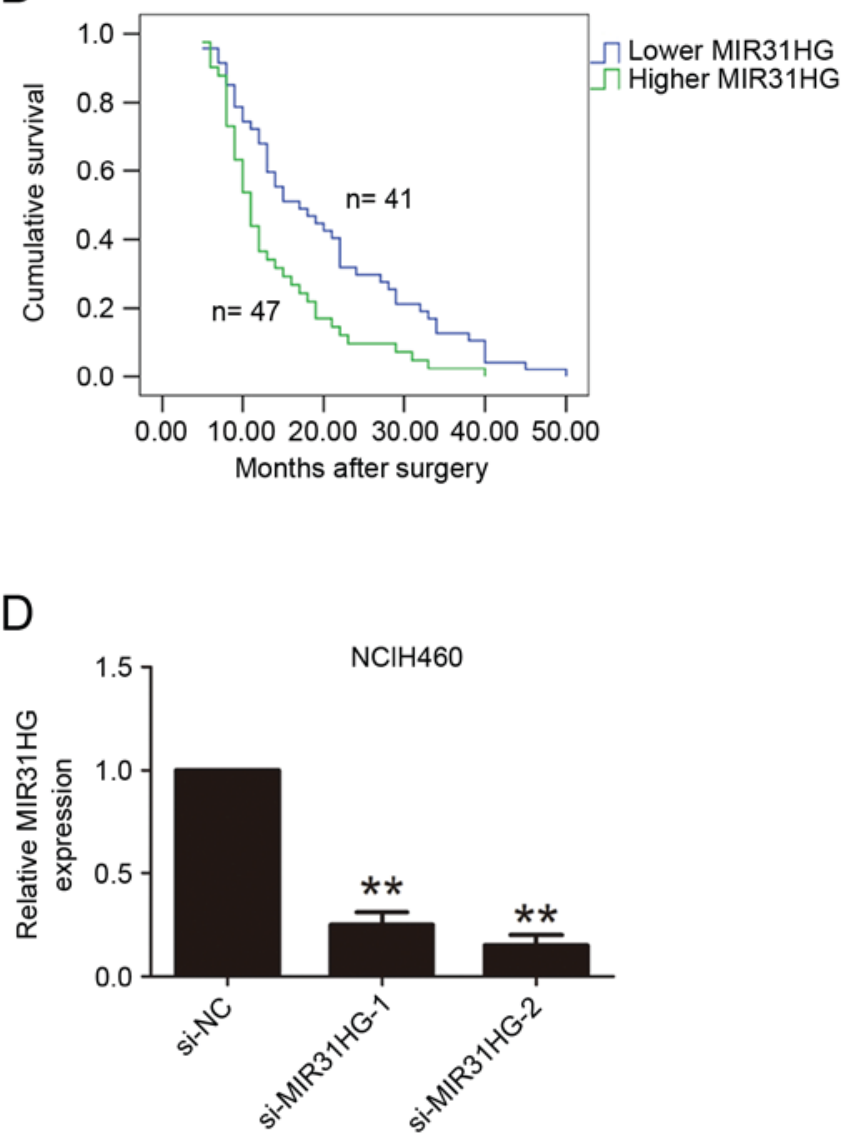

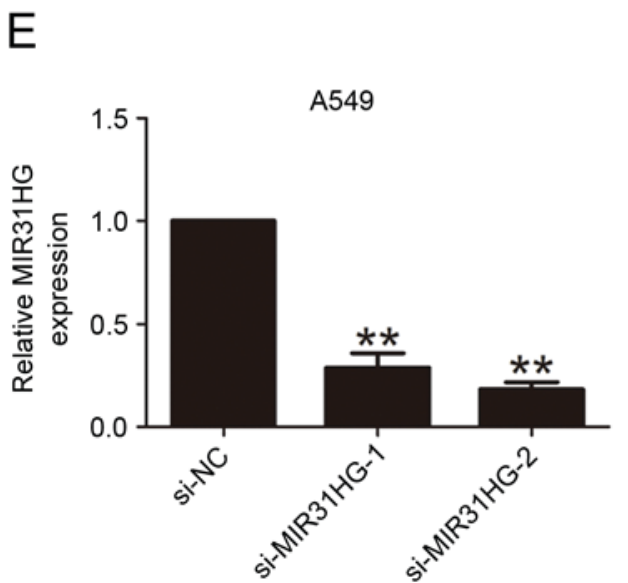

Figure 1. MIR31HG has higher expression in NSCLC tissues and cells. (A) RT-qPCR analysis of MIR31HG expression in 88 cases of NSCLC tissues and adjacent normal tissues. The expression of MIR31HG was normalized to GAPDH expression. (B) Kaplan-Meier survival analysis was performed to detect the association between MIR31HG expression and clinicopathologic parameters in 88 cases of NSCLC patients. (C) RT-qPCR analysis of MIR31HG expression in the three human NSCLC cell lines A549, H1299 and NCIH460 and the normal human bronchial epithelial cell line 16HBE. The expression of MIR31HG was normalized to GAPDH expression. RT-qPCR analysis of MIR31HG expression following transfection with si-NC, si-MIR31HG-1, si-MIR31HG-2 into (D) NCIH460 or (E) A549 cells, The expression of MIR31HG was normalized to GAPDH expression. ${ }^{* *} \mathrm{P}<0.05$ vs. non-tumor, $16 \mathrm{HBE}$, si-NC. Every independent experiment was performed three times. NSCLC, non-small cell lung cancer; RT-qPCR, reverse transcription-quantitative polymerase chain reaction; si, small interfering; $\mathrm{NC}$, negative control.

\section{Results}

MIR3IHG has a higher expression in NSCLC tissues and cells. RT-qPCR was applied to determine the expression levels of MIR31HG in 88 cases of tumor tissues and adjacent normal tissues in patients with NSCLC. As demonstrated in
Fig. 1, MIR31HG was highly expressed in tumor tissues, when compared with adjacent normal tissues $(\mathrm{P}<0.05)$. Furthermore, the present study evaluated whether MIR31HG expression was correlated with clinicopathological factors in patients. The patients with NSCLC were classified into two groups, comprised of the higher expression and lower expression group, according 
A

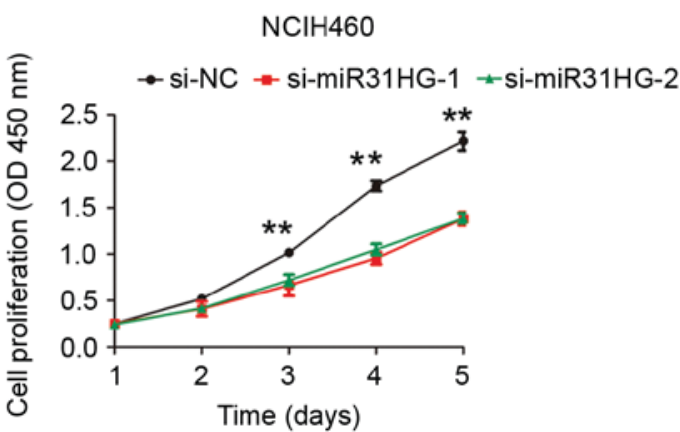

C
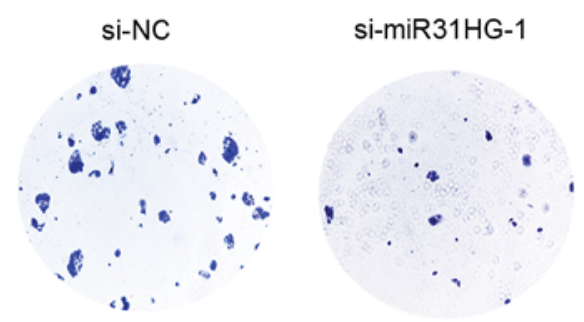

$\mathrm{NClH} 460$

E

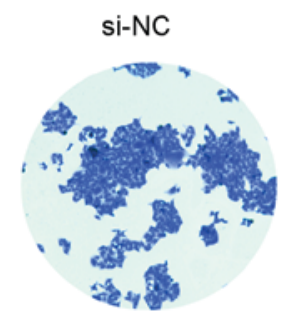

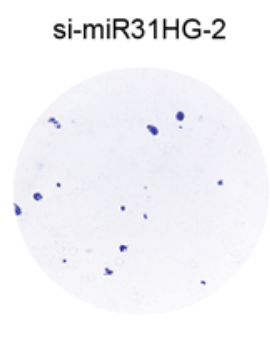

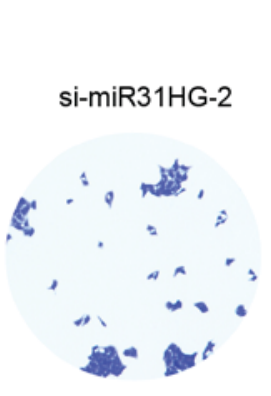

B

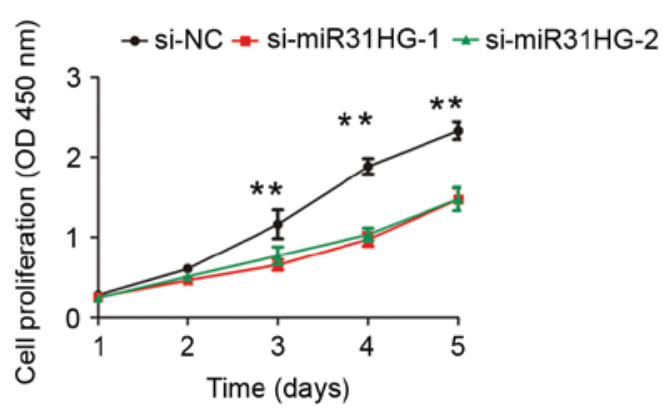

D

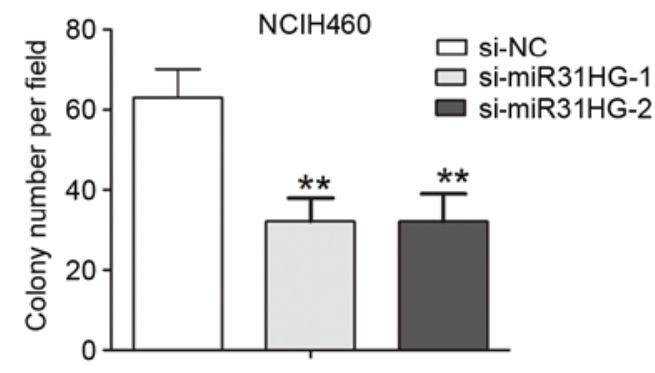

F

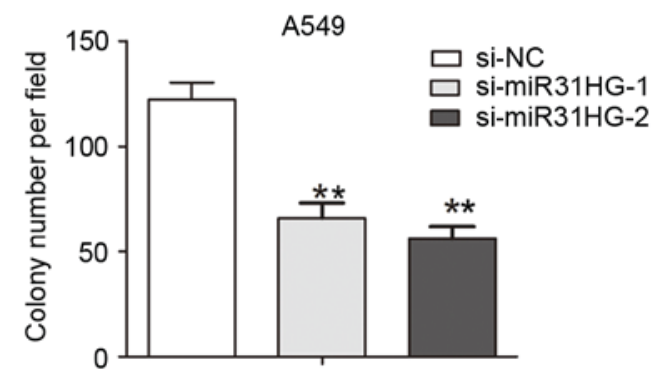

Figure 2. Knockdown of MIR31HG suppressed cell proliferation in NSCLC cells. (A) NCIH460 or (B) A549 cells were transfected with si-NC, si-MIR31HG-1, si-MIR31HG-2 and cell proliferation was determined using CCK8 assays. (C) NCIH460 cells were transfected with si-NC, si-MIR31HG-1, si-MIR31HG-2 and cell colony formation assay was performed, and (D) quantified. (E) A549 cells were transfected with si-NC, si-MIR31HG-1, si-MIR31HG-2 and cell colony formation assay was performed, and $(\mathrm{F})$ quantified. ${ }^{* *} \mathrm{P}<0.05 \mathrm{vs}$. si-NC. Every independent experiment was performed three times. NSCLC, non-small cell lung cancer; si, small interfering; NC, negative control; OD, optical density.

to the median value (2.68) of relative MIR31HG expression. The results demonstrated that the expression of MIR31HG was closely associated with histological differentiation grade $(\mathrm{P}=0.024)$, lymph node metastasis $(\mathrm{P}=0.021)$ and TNM stage $(\mathrm{P}=0.002)$ (Table I). Furthermore, Kaplan-Meier curves and log-rank tests were performed to analyze the prognostic value of MIR31HG expression for the OS time in patients with NSCLC. The results demonstrated that patients with higher MIR31HG expression have a poor OS time $(\mathrm{P}<0.05$, Fig. $1 \mathrm{~B})$. In addition, MIR31HG expression levels were detected in the three human NSCLC cells A549, H1299 and NCIH460, and a normal human bronchial epithelial cell line 16HBE, the results confirmed that MIR31HG expression levels were higher in NSCLC cells compared with that in $16 \mathrm{HBE}$ cells (Fig. 1C).

Decreased MIR31HG expression suppresses cell proliferation and migration ability in NSCLC cells. To evaluate the effects of MIR31HG expression on NSCLC cell proliferation and migration ability, two siRNA oligonucleotides against MIR31HG were used to knockdown MIR31HG in NCIH460 and A549 cells. The results revealed that MIR31HG was efficiently knocked-down following transfection with siRNA-MIR31HG-1 or siRNA-MIR31HG-2 into NCIH460 or A549 cells (Fig. 1D and E). CCK-8 cell proliferation assays demonstrated that the cell proliferation ability was inhibited following MIR31HG silencing in NCIH460 or A549 cells, compared with the si-NC group (Fig. 2A and B). The cell colony formation number was significantly decreased following MIR31HG silencing in NCIH460 or A549 cells compared with the si-NC group (Fig. 2C-F). Transwell cell invasion assays analysis demonstrated that cell invasion ability in NCIH460 or A549 cells was also inhibited following MIR31HG silencing compared with the si-NC group (Fig. 3). Taken together, these results confirmed 
A
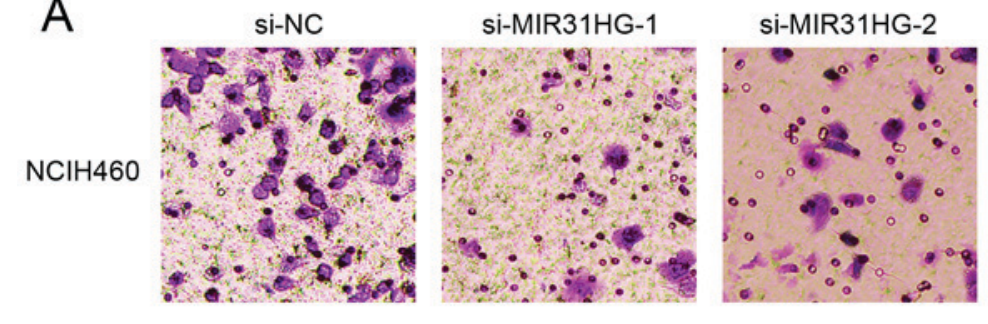

C

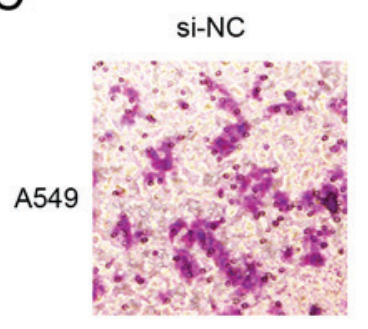

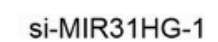

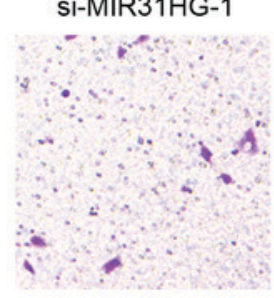

si-MIR31HG-2

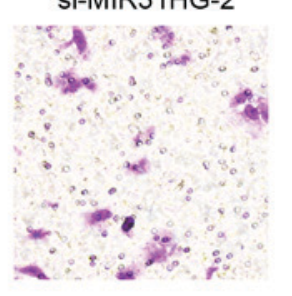

B

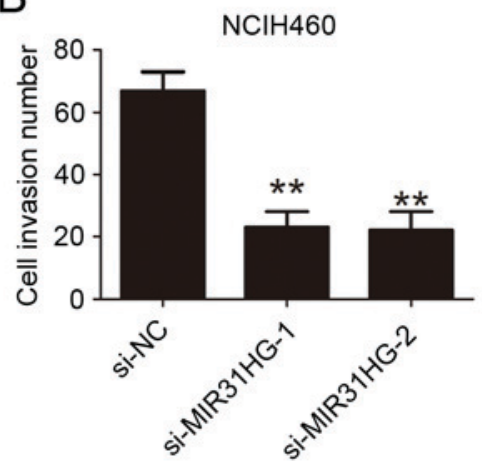

D

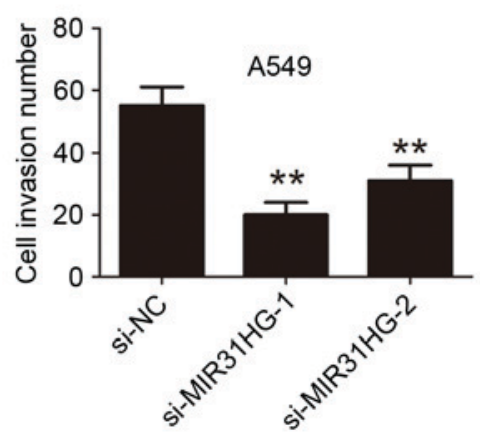

Figure 3. Knockdown of MIR31HG inhibited cell invasion in NSCLC cells. (A) NCIH460 cells were transfected with si-NC, si-MIR31HG-1, si-MIR31HG-2 and cell invasion ability was determined and (B) quantified using Transwell invasion assays. (C) A549 cells were transfected with si-NC, si-MIR31HG-1, si-MIR31HG-2 and cell invasion ability was determined and (D) quantified using Transwell invasion assays. ${ }^{* * *} \mathrm{P}<0.05$ vs. si-NC. Every independent experiment was performed three times. NSCLC, non-small cell lung cancer; si, small interfering; NC, negative control.

that MIR31HG promoted cell proliferation and invasion ability in NSCLC.

Knockdown of MIR31HG inhibits cell EMT and Wnt/ $\beta$-catenin signaling pathway in NSCLC cells. Cell invasion was associated with tumor EMT process, to explore whether MIR31HG affected cell EMT in NSCLC, the RT-qPCR and Western blotting assays were performed. The results showed that the mRNA expression levels of EMT related transcription factor Twist1 and mesenchymal marker Vimentin were significantly decreased, but epithelial marker E-cadherin was dramatically increased after MIR31HG silencing in NCIH460 or A549 cells (Fig. 4A and B). Furthermore, we detected their protein expression and the results demonstrated that protein expression of Twist1 and Vimentin were upregulated, but E-cadherin was downregulated after MIR31HG silencing in NCIH460 or A549 cells (Fig. $4 \mathrm{C}$ and $\mathrm{D}$ ). Wnt/ $\beta$-catenin signaling is associated with cell invasion and EMT process (14). Furthermore, the present study demonstrated that downregulated MIR31HG inhibited the Wnt/ $\beta$-catenin signaling pathway by decreasing the expression of GSK $3 \beta$ and $\beta$-catenin, but increasing the expression of p-GSK3 3 in NCIH460 or A549 cells (Fig. 5A and B). Therefore, these results indicated that MIR31HG promoted EMT by regulating the Wnt/ $\beta$-catenin signaling pathway in NSCLC cells.

\section{Discussion}

The precise molecular mechanism involved in lung carcinogenesis, at present, has not been completely elucidated. Recent studies provided the evidence that lncRNAs acted as prognostic predictor in lung cancer $(15,16)$, including increased expression of the long non-coding RNA ANRIL promoting lung cancer cell metastasis and was significantly correlated with poor prognosis (15). A previous study reported that growth arrest specific 5 (GAS5) expression was decreased in NSCLC plasma and the circulating long non-coding RNA GAS5 was a novel biomarker for the diagnosis of NSCLC (16). The expression level of tumor suppressor candidate 7 (TUSC7) was lower in NSCLC tissues and low expression of TUSC7 was an independent poor prognostic indicator for patients with NSCLC (17). Increased serum XIST and HIF1A-AS1 could be used as a predictive biomarker for NSCLC screening, and combination of XIST and HIF1A-AS1 showed a higher positive diagnostic efficiency of NSCLC (18). In the present study, it was revealed that MIR31HG was significantly increased in NSCLC tissues, when compared with adjacent normal tissues. Compared with lower MIR31HG in patients, higher MIR31HG predicted a poor prognosis for patients with NSCLC.

EMT is a cellular process that is involved in embryonic development and is classically characterized by the dedifferentiation from an epithelial to mesenchymal phenotype (19). The EMT phenotype is marked by the downregulated expression of E-cadherin and upregulated expression of $\mathrm{N}$-cadherin, vimentin and culminates in the higher expression of the transcription factors Snail, Zeb, Twist $(20,21)$. HNF1A-AS1 was revealed to promote tumor proliferation and metastasis, in vitro and in vivo, by regulating the expression of cyclin $\mathrm{D} 1, \mathrm{E}$-cadherin, $\mathrm{N}$-cadherin and $\beta$-catenin (22). A previous 
A

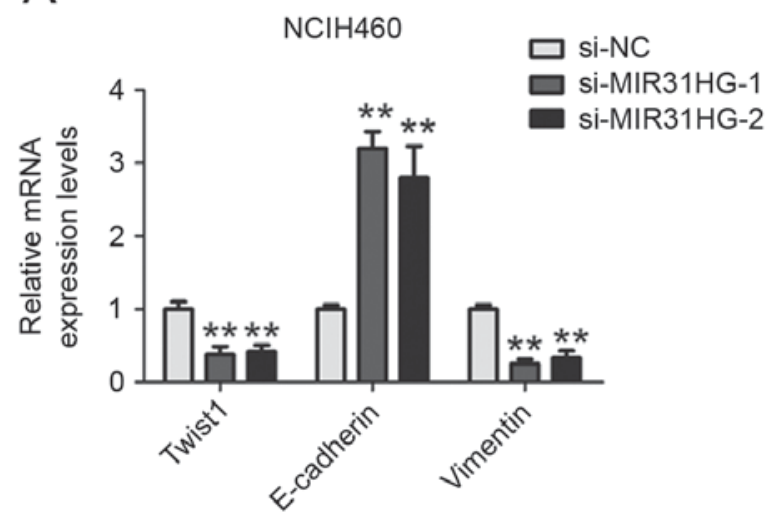

C

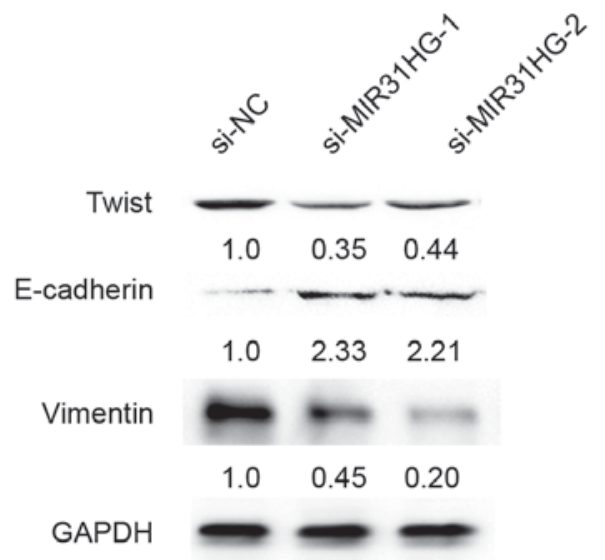

$\mathrm{NClH} 460$
B

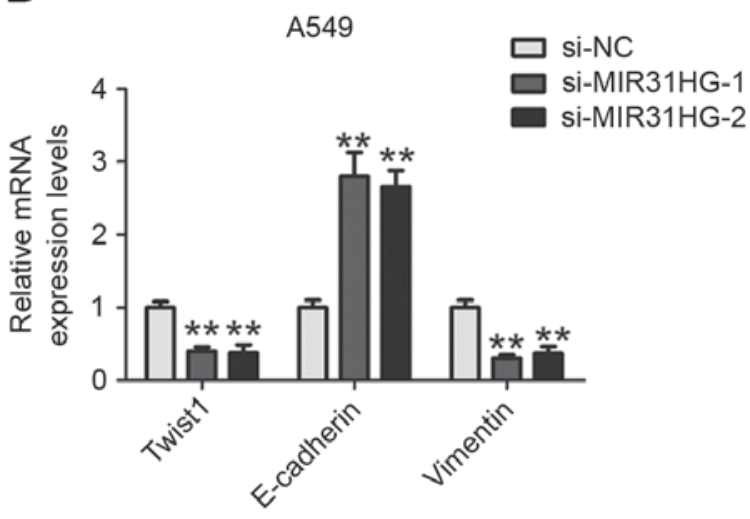

D

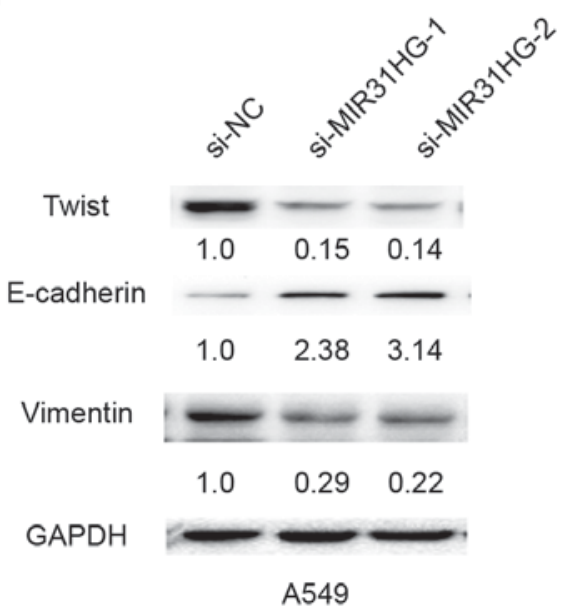

Figure 4. Knockdown of MIR31HG inhibited EMT in NSCLC cells. RT-qPCR analysis of Twist1, E-cadherin and Vimentin expression levels following transfection with si-NC, si-MIR31HG-1, si-MIR31HG-2 into (A) NCIH460 or (B) A549 cells, The expression of mRNA was normalized to GAPDH expression. Western blot analysis of Twist1, E-cadherin and Vimentin expression levels after transfected with si-NC, si-MIR31HG-1, si-MIR31HG-2 into (C) NCIH460 or (D) A549 cells. The numbers present the protein expression levels normalized to GAPDH expression. ${ }^{* *} \mathrm{P}<0.05$ vs. si-NC. Every independent experiment was performed three times. EMT, epithelial-mesenchymal transition; NSCLC, non-small cell lung cancer; si, small interfering; NC, negative control.

A

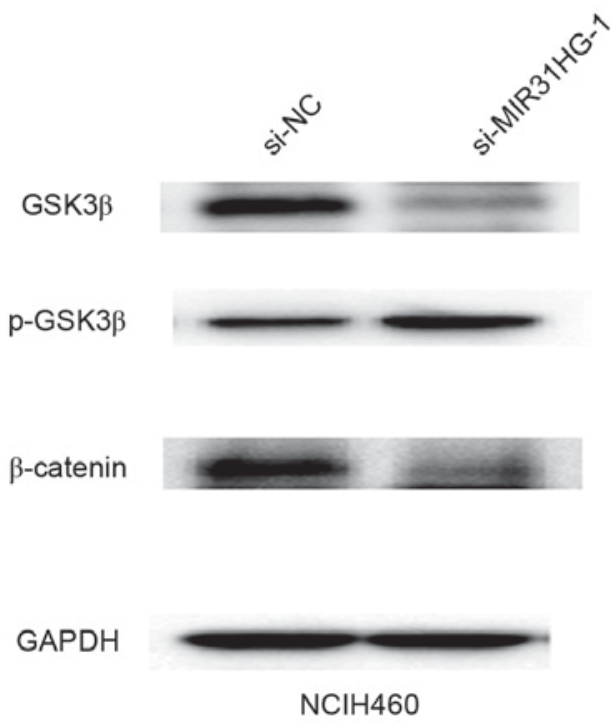

B

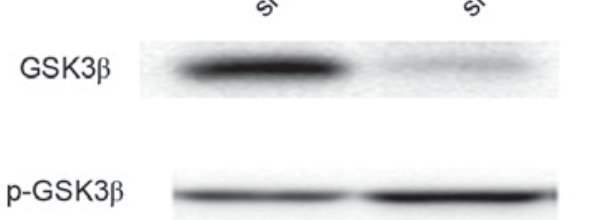

$\beta$-catenin

GAPDH

A549

Figure 5. Knockdown of MIR31HG inhibited the Wnt/ $\beta$-catenin signaling pathway in NSCLC cells. (A) The relative protein expression levels of GSK3 $\beta$, p-GSK $3 \beta$ and $\beta$-catenin were detected by western blot analysis following transfection with si-MIR31HG-1 into NCIH460 cells. (B) The relative protein expression levels of GSK3 $\beta$, p-GSK3 $\beta$ and $\beta$-catenin were detected by western blot analysis following transfection with si-MIR31HG-1 into A549 cells. Every independent experiment was performed three times. NSCLC, non-small cell lung cancer; si, small interfering; NC, negative control; p, phosphorylated; GSK $\beta$, glycogen synthase kinase $3 \beta$. 
study demonstrated that the loss of the 1ncRNA FOXF1-AS1 regulated EMT, stemness and metastasis of NSCLC cells (23). Downregulation of 1ncRNA BRAF activated non-coding RNA and was associated with poor prognosis for NSCLC and promoted metastasis by affecting EMT (24). LncRNA BC087858 induced non-T790M mutation and acquired resistance to EGFR-TKIs by activating PI3K/AKT and MEK/ERK pathways and EMT in NSCLC (25). In the present study, it was demonstrated that the knockdown of MIR31HG inhibited the cell proliferation, migration and EMT in NSCLC cells by downregulating the expression of Twist1 and Vimentin and upregulating the expression of E-cadherin.

Wnt/ $\beta$-catenin signaling has been reported to be involved in NSCLC invasion and metastatic abilities (14). For example, Astrocyte elevated gene-1 (AEG-1) induced EMT in lung cancer through activating Wnt/ $\beta$-catenin signaling (26). Cucurbitacin B inhibits the stemness and metastatic abilities of NSCLC via downregulation of the Wnt/ $\beta$-catenin signaling axis (27). In the present study, downregulated MIR31HG was demonstrated to inhibit the $\mathrm{Wnt} / \beta$-catenin signaling pathway by decreasing the expression of GSK $3 \beta$ and $\beta$-catenin, but increasing the $\mathrm{p}-\mathrm{GSK} 3 \beta$ expression in NSCLC cells. The limitation of the present study is that it was only demonstrated that downregulated MIR31HG inhibited the Wnt/ $\beta$-catenin signaling pathway. The effects of MIR31HG expression on other signaling pathways requires further investigated. The investigation of molecular mechanism underlying NSCLC cell proliferation and invasion for MIR31HG may provide a potential target for NSCLC therapy in the future.

To conclude, the results of the present study demonstrated that MIR31HG was significantly increased in NSCLC tissues and increased MIR31HG predicted a poor survival time in patients with NSCLC. Furthermore, the downregulation of MIR31HG significantly inhibited NSCLC cell proliferation, cell invasion and EMT phenotype. Additionally, downregulated MIR31HG inhibited the Wnt/ $\beta$-catenin signaling pathway. These results demonstrated that MIR31HG could be identified as a poor prognostic biomarker and a therapeutic target for patients with NSCLC.

\section{Acknowledgements}

Not applicable.

\section{Funding}

No funding was received.

\section{Availability of data and materials}

The datasets used and/or analyzed during the current study are available from the corresponding author on reasonable request.

\section{Authors' contributions}

$\mathrm{SZ}$ and $\mathrm{XZ}$ designed the study. SZ and $\mathrm{XZ}$ performed the experiments. XW and JL analyzed the data. All authors collaborated to interpret results and develop the manuscript. The final version of the manuscript has been read and approved by all authors.

\section{Ethics approval and consent to participate}

The study was approved by the Ethics Committee of The First Affiliated Hospital and College of Clinical Medicine of Henan University of Science and Technology (Luoyang, China). Written, informed consent was obtained from all patients.

\section{Patient consent for publication}

Written informed consent was obtained from all patients.

\section{Competing interests}

The authors declare that they have no competing interests.

\section{References}

1. Crinò L, Weder W, van Meerbeeck J and Felip E; ESMO Guidelines Working Group: Early stage and locally advanced (non-metastatic) non-small-cell lung cancer: ESMO Clinical Practice Guidelines for diagnosis, treatment and follow-up. Ann Oncol 21 (Suppl 5): v103-v115, 2010.

2. Hackner K, Errhalt P, Mueller MR, Speiser M, Marzluf BA, Schulheim A, Schenk P, Bilek J and Doll T: Canine scent detection for the diagnosis of lung cancer in a screening-like situation. J Breath Res 10: 046003, 2016.

3. Boffa DJ, Allen MS, Grab JD, Gaissert HA, Harpole DH and Wright CD: Data from The Society of Thoracic Surgeons General Thoracic Surgery database: The surgical management of primary lung tumors. J Thorac Cardiovasc Surg 135: 247-254, 2008.

4. Esteller M: Non-coding RNAs in human disease. Nat Rev Genet 12: 861-874, 2011.

5. Chen J, Wang R, Zhang K and Chen LB: Long non-coding RNAs in non-small cell lung cancer as biomarkers and therapeutic targets. J Cell Mol Med 18: 2425-2436, 2014.

6. Mercer TR, Dinger ME and Mattick JS: Long non-coding RNAs: Insights into functions. Nat Rev Genet 10: 155-159, 2009.

7. Montes M, Nielsen MM, Maglieri G, Jacobsen A, Højfeldt J, Agrawal-Singh S, Hansen K, Helin K, van de Werken HJ, Pedersen JS and Lund AH: The lncRNA MIR31HG regulates p16(INK4A) expression to modulate senescence. Nat Commun 6: $6967,2015$.

8. Nie FQ, Ma S, Xie M, Liu YW, De W and Liu XH: Decreased long noncoding RNA MIR31HG is correlated with poor prognosis and contributes to cell proliferation in gastric cancer. Tumour Biol 37: 7693-7701, 2016.

9. He A, Chen Z, Mei H and Liu Y: Decreased expression of LncRNA MIR31HG in human bladder cancer. Cancer Biomark 17: 231-236, 2016.

10. Yang H, Liu P, Zhang J, Peng X, Lu Z, Yu S, Meng Y, Tong WM and Chen J: Long noncoding RNA MIR31HG exhibits oncogenic property in pancreatic ductal adenocarcinoma and is negatively regulated by miR-193b. Oncogene 35: 3647-3657, 2016.

11. Wang B, Jiang H, Wang L, Chen X, Wu K, Zhang S, Ma S and Xia B: Increased MIR31HG lncRNA expression increases gefitinib resistance in non-small cell lung cancer cell lines through the EGFR/PI3K/AKT signaling pathway. Oncol Lett 13: 3494-3500, 2017

12. Kaur H, Sehgal IS, Bal A, Gupta N, Behera D, Das A and Singh N: Evolving epidemiology of lung cancer in India: Reducing non-small cell lung cancer-not otherwise specified and quantifying tobacco smoke exposure are the key. Indian J Cancer 54: 285-290, 2017.

13. Livak and Schmittgen: Analysis of relative gene expression data using real-time quantitative PCR and the 2(-Delta Delta C(T)) method. Methods 25: 402-408, 2001.

14. Kahlert UD, Nikkhah G and Maciaczyk J: Epithelial-tomesenchymal(-like) transition as a relevant molecular event in malignant gliomas. Cancer Lett 331: 131-138, 2013.

15. Lin L, Gu ZT, Chen WH and Cao KJ: Increased expression of the long non-coding RNA ANRIL promotes lung cancer cell metastasis and correlates with poor prognosis. Diagn Pathol 10: $14,2015$. 
16. Liang W, Lv T, Shi X, Liu H, Zhu Q, Zeng J, Yang W, Yin J and Song Y: Circulating long noncoding RNA GAS5 is a novel biomarker for the diagnosis of nonsmall cell lung cancer. Medicine (Baltimore) 95: e4608, 2016.

17. Wang $Z$, Jin $Y$, Ren $H$, Ma X, Wang $B$ and Wang $Y$ : Downregulation of the long non-coding RNA TUSC7 promotes NSCLC cell proliferation and correlates with poor prognosis Am J Transl Res 8: 680-687, 2016.

18. Tantai J, Hu D, Yang Y and Geng J: Combined identification of long non-coding RNA XIST and HIF1A-AS1 in serum as an effective screening for non-small cell lung cancer. Int J Clin Exp Pathol 8: 7887-7895, 2015.

19. Micalizzi DS and Ford HL: Epithelial-mesenchymal transition in development and cancer. Future Oncol 5: 1129-1143, 2009.

20. Felipe Lima J, Nofech-Mozes S, Bayani J and Bartlett JM: EMT in breast carcinoma-A review. J Clin Med 5: pii: E65, 2016.

21. Prat A and Perou CM: Deconstructing the molecular portraits of breast cancer. Mol Oncol 5: 5-23, 2011.

22. Wu Y, Liu H, Shi X, Yao Y, Yang W and Song Y: The long non-coding RNA HNF1A-AS1 regulates proliferation and metastasis in lung adenocarcinoma. Oncotarget 6: 9160-9172, 2015.

23. Miao L, Huang Z, Zengli Z, Li H, Chen Q, Yao C, Cai H, Xiao Y, $\mathrm{Xia} \mathrm{H}$ and Wang Y: Loss of long noncoding RNA FOXF1-AS1 regulates epithelial-mesenchymal transition, stemness and metastasis of non-small cell lung cancer cells. Oncotarget 7: 68339-68349, 2016.
24. Sun M, Liu XH, Wang KM, Nie FQ, Kong R, Yang JS, Xia R, $\mathrm{Xu}$ TP, Jin FY, Liu ZJ, et al: Downregulation of BRAF activated non-coding RNA is associated with poor prognosis for non-small cell lung cancer and promotes metastasis by affecting epithelial-mesenchymal transition. Mol Cancer 13: 68, 2014.

25. Pan H, Jiang T, Cheng N, Wang Q, Ren S, Li X, Zhao C, Zhang L, Cai W and Zhou C: Long non-coding RNA BC087858 induces non-T790M mutation acquired resistance to EGFR-TKIs by activating PI3K/AKT and MEK/ERK pathways and EMT in non-small-cell lung cancer. Oncotarget 7: 49948-49960, 2016.

26. He W, He S, Wang Z, Shen H, Fang W, Zhang Y, Qian W, Lin M, Yuan J, Wang J, et al: Astrocyte elevated gene-1 (AEG-1) induces epithelial-mesenchymal transition in lung cancer through activating Wnt/ $\beta$-catenin signaling. BMC Cancer 15: 107, 2015.

27. Shukla S, Sinha S, Khan S, Kumar S, Singh K, Mitra K, Maurya R and Meeran SM: Cucurbitacin B inhibits the stemness and metastatic abilities of NSCLC via downregulation of canonical Wnt//3-catenin signaling axis. Sci Rep 6: 21860, 2016.

cc) (7) (5) This work is licensed under a Creative Commons Attribution 4.0 International (CC BY-NC 4.0) License 